\title{
Cancer stem cell associated eight gene-based signature predicts clinical outcomes of colorectal cancer
}

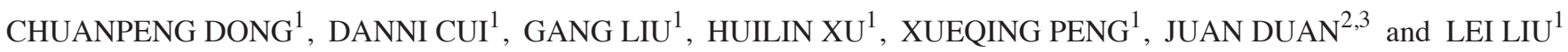 \\ ${ }^{1}$ Institute of Biomedical Sciences, Fudan University, Shanghai 200032; ${ }^{2}$ Department of Biochemistry and \\ Molecular Biology, School of Basic Medical Sciences; ${ }^{3}$ Translational Medicine Institute, \\ Fujian Medical University, Fuzhou, Fujian 350004, P.R. China
}

Received July 7, 2016; Accepted July 20, 2017

DOI: $10.3892 / \mathrm{ol} .2018 .9533$

\begin{abstract}
Previous studies have suggested that cancer stem cells serve crucial functions in tumorigenesis, metastasis and therapy failure. Stem cell signaling transduction pathways are frequently dysregulated in cancer and associated with tumorigenesis, metastasis and the cell cycle, which are necessary for cancer proliferation. However, cancer stem cell-associated gene signatures have not been established for predicting patient outcomes in colorectal cancer. Using a gene-mining approach, the present study performed mRNA expression profiling in large colorectal cancer cohorts from The Cancer Genome Atlas (TCGA) and the Gene Expression Omnibus (GEO) database, including a TCGA colorectal cancer cohort $(n=383)$ and three independent validation series GSE39582 $(n=582), \operatorname{GSE} 17536(n=177)$ and GSE17537 $(n=55)$. The present study identified that an eight-gene signature in cancer stem cell signaling was associated with the overall survival and disease/recurrence-free survival of patients with colorectal. On the basis of this signature, patients in the TCGA training sets were divided into high-risk and low-risk subgroups with a significantly different overall survival rate (hazard ratio, 2.38; $\mathrm{P}=0.0005$ ). The prognostic value of this signature was confirmed using three independent GEO colorectal cancer sets. Identifying this prognostic stem cell signaling signature may provide an efficient classification tool for clinical prognosis evaluation, and facilitate cancer stem cell-targeted therapy.
\end{abstract}

\section{Introduction}

Colorectal cancer is one of the most common types of cancer and one of the leading causes of cancer-associated mortality globally, according to statistical data in 2013 (1). Identifying cancer stem-like cells (CSCs) may be essential for improving

Correspondence to: Dr Lei Liu, Institute of Biomedical Sciences, Fudan University, 131 Dongan Road, Shanghai 200032, P.R. China E-mail: liulei_fdu@163.com

Key words: gene signature, cancer stem cell, colorectal cancer, prognosis targeted cancer therapy $(2,3)$. However, detecting purified CSCs in colorectal cancer remains challenging with existing methods, since few specific markers are known for CSCs in colorectal cancer $(4,5)$.

CSCs may trigger tumorigenesis, self-renewal, differentiation and resistance to therapy (6). Previous studies have demonstrated that CSCs may activate one or more highly conserved signaling pathways present in normal stem cells that are associated with development and tissue homeostasis, including the Notch (7), Hedgehog (8) and Wnt (9) signaling pathways. These pathways are associated with tumorigenesis, metastasis and the cell cycle, which are required for cancer proliferation (10). Crosstalk between signaling pathways also increases the complexity of cellular external stimuli response networks (11). Upregulating secreted frizzled related protein (SFRP)1 expression in the Hedgehog signaling pathway inhibited the Wnt signaling pathway (12), whereas activating the Hedgehog signaling pathway resulted in increased jagged 2 expression and the upregulation of the Notch signaling pathway (11). A network-level view of signaling pathways in cancer stem cells is required to identify shared features among malignant cells and provide means for clinical therapy to develop.

Previously, multiple studies have developed multigene classifiers for determining the prognosis of patients with colon cancer, including the 12-gene based Oncotype DX $(13,14)$, 18-gene based ColoPrint (15) and the 13-gene based classifier ColoGuideEx (16). Compared with conventional pathological criteria alone, genomic classifiers, including Oncotype DX, Coloprint and ColoGuideEx, provide more accurate information on the risk of recurrence and may assist in selecting patients who can benefit more from chemotherapy. However, these gene-based classification systems may not assist in developing a patient selection tool for specific inhibitor-targeted therapy. Therefore, establishing a signaling pathway- or multiple signaling pathways-based gene expression signature to facilitate treatment decisions is crucial.

The present study assessed the cancer stem cell signaling pathways and the gene expression profiles of 1,198 patients with colorectal cancer from The Cancer Genome Atlas (TCGA) and the Gene Expression Omnibus (GEO) database. By analyzing the association between gene expression profiling and the clinical outcome of patients with colorectal cancer, 
the present study identified an eight-gene signature associated with the Hedgehog-Notch-Wnt signaling pathways, which was associated with the prognosis of the patients. The results of the present study may assist in developing therapeutic strategies for treating colorectal cancer.

\section{Materials and methods}

Colorectal cancer data. Colorectal cancer gene expression datasets and the corresponding clinical data were downloaded from TCGA and the GEO database. Integrated gene expression and clinical data of TCGA were downloaded from the University of California, Santa Cruz Cancer Genomics Browser (Santa Cruz, CA, USA; https://genome-cancer.ucsc.edu) (17). In the present study, 383 colorectal tumors from patients with detailed gene expression information were chosen from TCGA according to parameters defined in a previous study (18); three datasets GSE39582 (n=582) (19), GSE17536 (n=177) (20) and GSE17537 $(n=55)$ (21) from the GEO database (http://www.ncbi.nlm.nih. gov/geo) were selected as testing datasets and contained overall survival and recurrence/disease-free survival information. A total of 1,198 patients were analyzed in the present study. Clinical information was extracted from the original publications (19-21). The workflow of the present study is presented in Fig. 1.

Candidate gene selection. The present study selected genes that are associated with the signaling or transcriptional regulators of CSCs in the Hedgehog, Notch and Wnt signaling pathways. To identify more genes associated with these signaling pathways, SABiosciences (http://www.sabiosciences.com/PCRArrayPlate. php) and polymerase chain reaction (PCR) array gene lists were used to form a gene list and group functional genes (22). The gene tables contained Hedgehog/Notch/Wnt ligands, receptors and regulators, and downstream signaling molecules and target proteins associated with these signaling pathways.

Statistical analysis. The association between gene expression and patient survival rate was assessed with univariate Cox's regression analysis and a permutation test using Biometric Research Branch (BRB)-Array Tools edition 4.5.0 (https://brb.nci.nih.gov/BRB-ArrayTools/) (23). Genes were considered statistically significant if their permutation P-value was $\leq 0.01$. Selected genes were fitted using a multivariable Cox regression model in the training set as described. A risk score formula was constructed by including statistically significant genes, weighted by their estimated Cox's regression coefficients (24). Patients with assigned risk score were classified into high-risk or low-risk groups by using the median as the threshold. Kaplan-Meier estimator survival analysis using the R package survival (v2.41-3; https://cran.r-project. org/web/packages/survival/) was performed to estimate the survival distributions between stratified survival groups in each set $(25,26)$. The two-sided log-rank test was used to assess the survival differences between high-risk and low-risk groups. A two-way ANOVA was used to analyze the association between the eight-gene signature and the American Joint Committee on Cancer stage.

The receiver operating characteristic (ROC) curve was constructed using the $\mathrm{R}$ package pROC to evaluate the sensitivity and specificity of the survival prediction for the
CSC signature risk score, age and ColoGuideEx, a reported prognostic predictor. According to ColoGuideEx, a 13-gene prognostic predictor developed by Agesen et al (16), patients were stratified according to the number of genes exceeding the $80 \%$ high-risk genes and below the $20 \%$ level of low-risk genes. Area under the curve values were calculated from the ROC curves.

\section{Results}

Identifying prognostic CSC-associated genes from the TCGA COADREAD dataset. A total of 334 CSC-associated genes in the Hedgehog, Notch and Wnt signaling pathways and their targets were identified from the colorectal tumors in the TCGA cohorts and the GEO datasets. The 383 TCGA patients cohort was defined as the training set, and was used to select the prognostic CSC-associated genes. Using BRB-Array Tools, univariate Cox's proportional hazards regression analysis was performed for the CSC-associated gene expression data, and eight CSC-associated genes were identified as significantly associated with overall survival $(\mathrm{P} \leq 0.01)$. Of these genes, a hazard ratio $>1$, which was associated with low-density lipoprotein-related protein 2 (LRP2), hairy/enhancer-of-split associated with YRPW motif-like protein (HEYL), cubilin (CUBN), SFRP2, growth arrest and DNA-damage-inducible $45 \beta$ (GADD45B), insulin-like growth factor-binding protein 3 (IGFBP3) and lymphoid enhancer-binding factor 1 (LEF1), indicated that high expression of that gene was associated with poor survival; a hazard ratio $<1$, which was associated with cyclin E1 (CCNE1), indicated that increased expression of that gene was associated with good survival (Table I).

Association of the eight-gene signature and patient survival in the training set. A risk-score formula was established according to the expression of these significant CSC-associated genes and their respective coefficients: Risk score $=0.253 \times \mathrm{L}$ $\mathrm{RP} 2+0.062 \times \mathrm{HEYL}+0.048 \times \mathrm{CUBN}+0.036 \times \mathrm{SFRP} 2+0$. $058 \times$ GADD $45 B+0.084 \times$ IGFBP3 + 0.139 x LEF1-0.190 x CCNE1. The present study calculated the eight-gene signature risk score for each patient in the training set. Patients with an assigned risk score were divided into high-risk or low-risk groups using the median risk score in the training set as the threshold. The distribution of patient risk scores, survival status and significant gene expression level were analyzed for the TCGA training set (Fig. 2). Patients in the high-risk group were associated with significantly decreased overall survival compared with patients in the low-risk group $(\mathrm{P}=0.00033$; Fig. 3A). The association of the eight-gene signature risk score with clinical outcome was significant when it was analyzed as a continuous variable in the univariate Cox's regression model: Overall survival (hazard ratio, 2.72; $\mathrm{P}<0.0001$ ) and recurrence-free survival (hazard ratio, 2.29; $\mathrm{P}<0.0001$ ).

Validating the eight-gene signature for survival prediction in the testing sets. To confirm the results of the present study, the risk score for the testing sets, including GSE39582, GSE17536 and GSE17537, were calculated. Similar to the TCGA training set, patients in the high-risk group were associated with decreased survival time compared with patients in the low-risk 
Table I. Eight genes associated with overall survival in the training-set patients.

Gene symbol

Full name

Parametric P-value Hazard ratio Coefficient

\begin{tabular}{|c|c|c|c|c|}
\hline LRP2 & Low-density lipoprotein-related protein 2 & 0.0005083 & 1.355 & 0.2530 \\
\hline HEYL & Hairy/enhancer-of-split associated with YRPW motif-like protein & 0.0011791 & 1.483 & 0.0617 \\
\hline CUBN & Cubilin & 0.0020518 & 1.461 & 0.0477 \\
\hline SFRP2 & Secreted frizzled-related protein 2 & 0.0025242 & 1.13 & 0.0360 \\
\hline GADD45B & Growth arrest and DNA-damage-inducible $45 \beta$ & 0.0033662 & 1.466 & 0.0582 \\
\hline IGFBP3 & Insulin-like growth factor-binding protein 3 & 0.0041609 & 1.352 & 0.0841 \\
\hline LEF1 & Lymphoid enhancer-binding factor 1 & 0.0044962 & 1.314 & 0.1389 \\
\hline CCNE1 & Cyclin E1 & 0.005372 & 0.627 & -0.1902 \\
\hline
\end{tabular}

Coefficient, coefficient in the multivariable Cox's regression analysis.

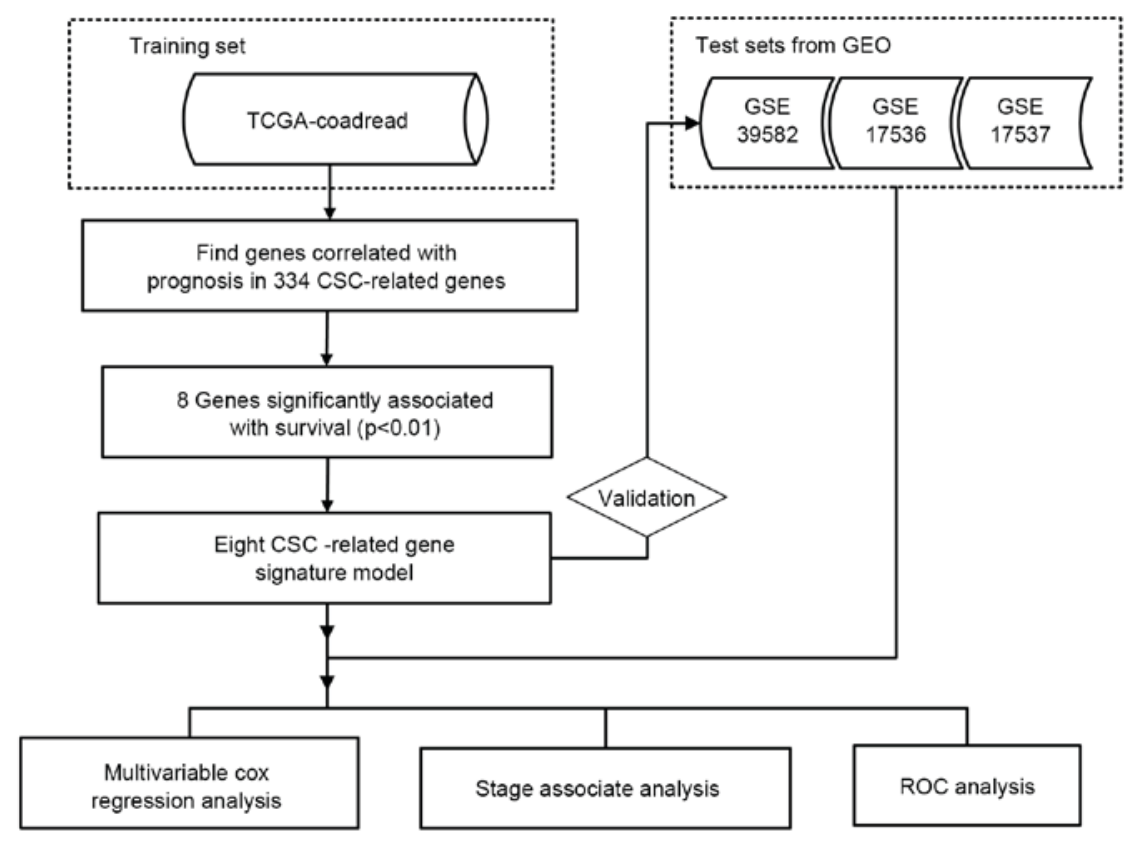

Figure 1. Workflow. TCGA, The Cancer Genome Atlas; GEO, Gene Expression Omnibus; CSC, cancer stem-like cell; ROC, receiver operating characteristic.

group (Fig. 3B-D). In addition, patient survival throughout the follow-up in the low-risk group was improved compared with that in the high-risk group. The univariate Cox's regression model revealed a similar association between risk score and overall survival, with the high-risk group associated with decreased overall survival compared with the low-risk group.

The prognostic value of the signature for the patients with or without post-operative chemotherapy was also assessed, according to the treatment records provided in the original raw data in GEO. Adjuvant chemotherapy information was available for the GSE39582 series; 240 patients received adjuvant chemotherapy, and 323 did not. The present study revealed that the high-risk score was significantly associated with unfavorable overall and recurrence-free survival in patients with or without post-operative chemotherapy (Fig. 4).

Eight-gene signature represents an independent predictor in colorectal cancer. The present study performed Cox's univariate and multivariate analysis to ascertain whether the eight-gene signature represented an independent predictor of overall survival in patients with colorectal cancer (Table II). The effect of risk score, age, sex and stage on patient survival time was analyzed further using a multivariate Cox's proportional hazard model for each cohort. The results indicated that risk score could represent an independent predictor of overall survival when adjusted for age, sex or stage in three cohorts. In GSE17537 ( $n=55)$, univariate Cox's analysis demonstrated that the eight-gene model was statistically significant for prognosis, although multivariate Cox's analysis revealed it was not.

The present study also analyzed the association between the eight-gene signature and the American Joint Committee on Cancer stage (27). Mean risk score increased with tumor malignance in the training and testing sets (Fig. 5). Therefore, the risk score model of the present study may facilitate stratifying patients with colorectal cancer.

Evaluating the risk score using ROC analysis. ROC analysis was performed to compare the sensitivity and specificity of 


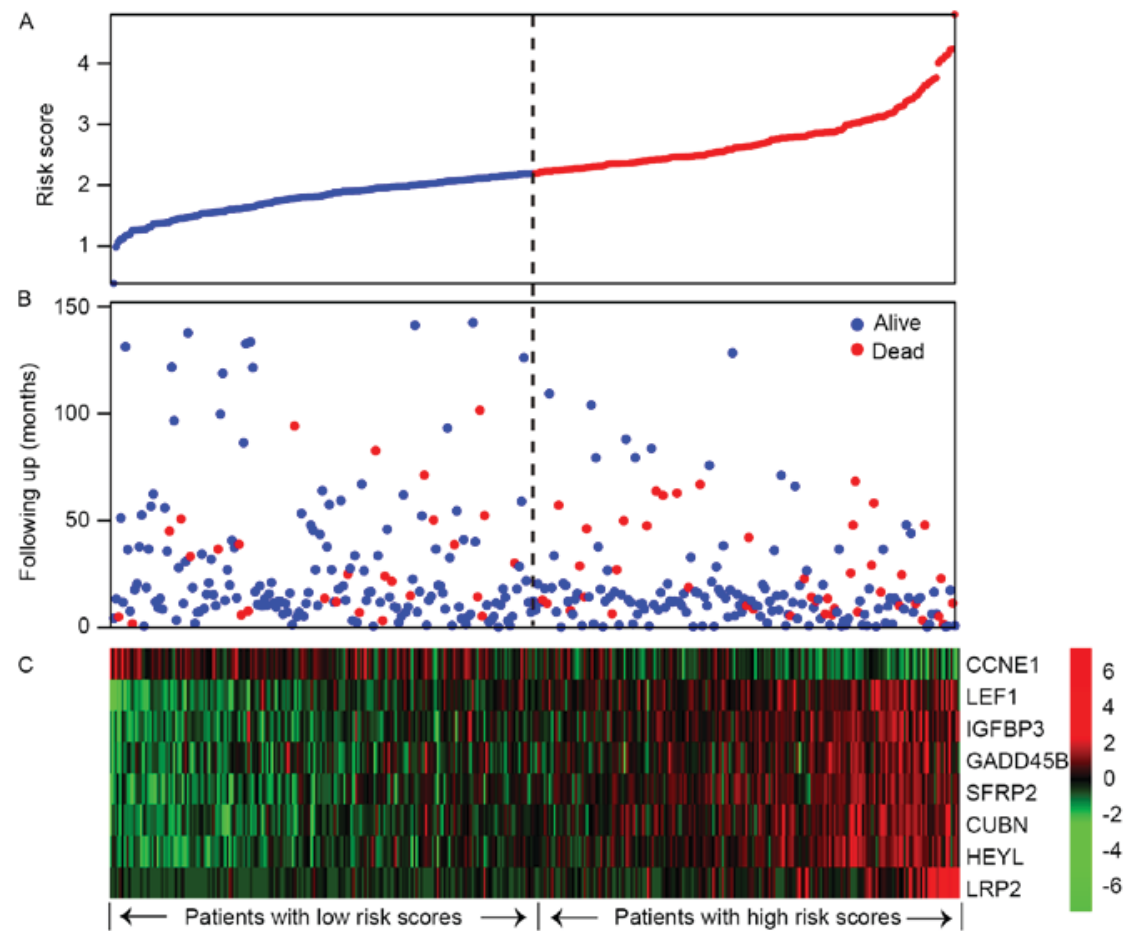

Figure 2. Eight gene-based risk score analysis of patient data from The Cancer Genome Atlas. The distributions of eight gene-based risk score, patient survival status and gene expression signature were analyzed for the training set patients $(n=364)$. (A) Eight gene-based risk score distribution. (B) Patient overall survival status and time. (C) Heat map of the eight gene expression profiles. Rows represent genes; columns represent patients. The black dotted line represents the risk score median threshold, which divided patients into low-risk and high-risk groups. CCNE1, cyclin E1; LEF1, lymphoid enhancer-binding factor 1; IGFBP3, insulin-like growth factor-binding protein 3; GADD45B, growth arrest and DNA-damage-inducible 45 $\beta$; SFRP2, secreted frizzled-related protein 2; CUBN, cubilin; HEYL, hairy/enhancer-of-split associated with YRPW motif-like protein; LRP2, low-density lipoprotein-related protein 2.
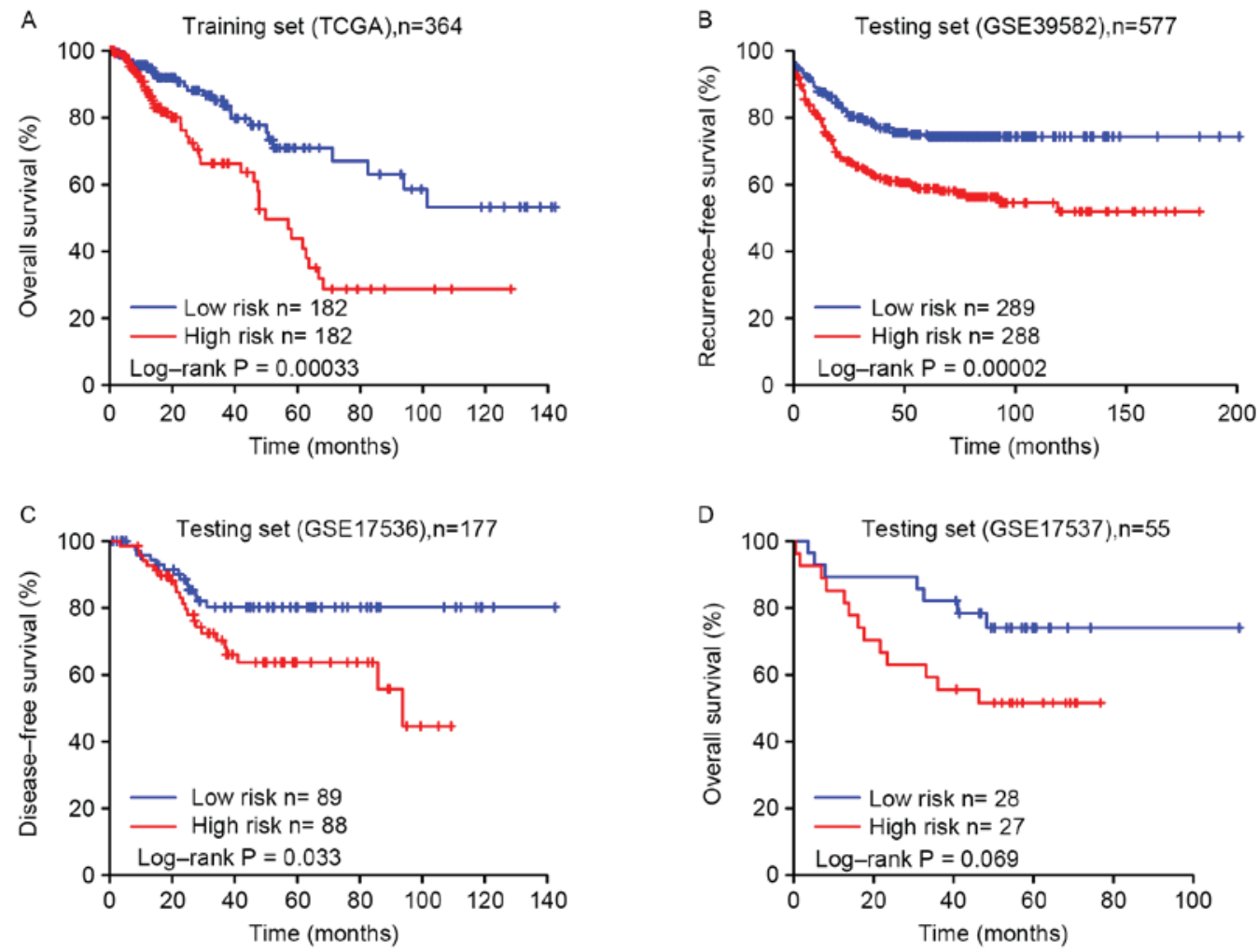

Figure 3. Kaplan-Meier estimates of the survival of patients using the eight-gene signature. The Kaplan-Meier estimator plots were used to visualize the survival probabilities for the low-risk and high-risk groups, as determined using the median risk score for each dataset. (A) Kaplan-Meier estimator curves of overall survival for TCGA training set patients $(\mathrm{n}=364)$. (B) Kaplan-Meier estimator curves of recurrence-free survival for GSE39582 testing set patients ( $\mathrm{n}=577$ ). (C) Kaplan-Meier estimator curves of disease-free survival for GSE17536 testing set patients ( $\mathrm{n}=177)$. (D) Kaplan-Meier estimator curves of overall survival for GSE17537 testing set patients n=55). Tick marks represent censored data (i.e., patients alive at the point of the last follow-up). The differences between the two curves were determined using the two-sided log-rank test. TCGA, The Cancer Genome Atlas. 
A

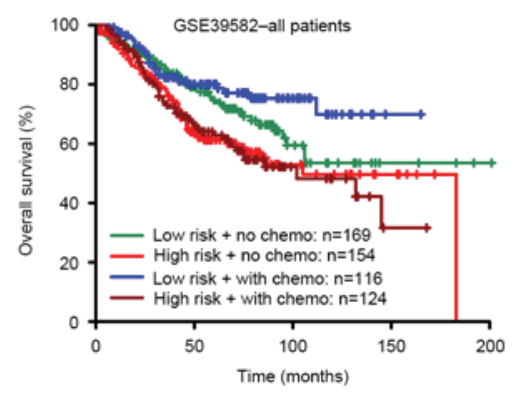

D

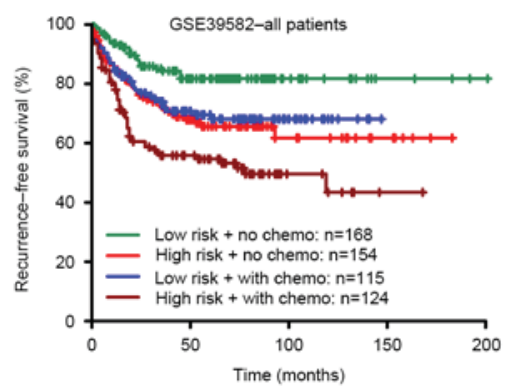

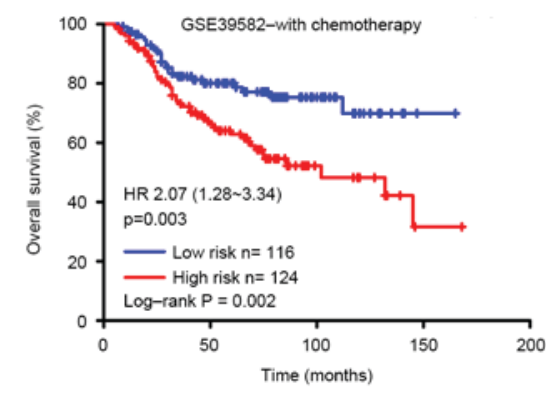

C

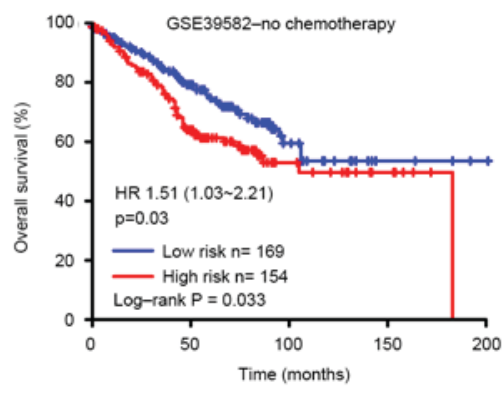

E

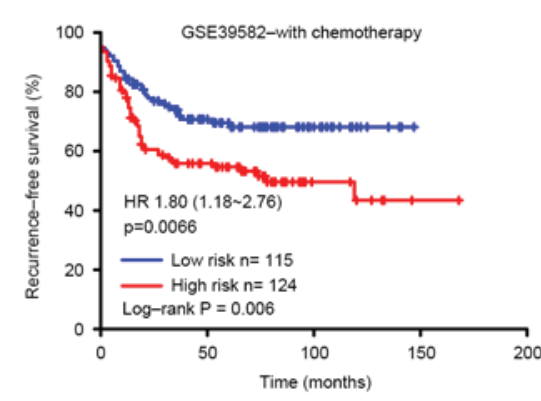

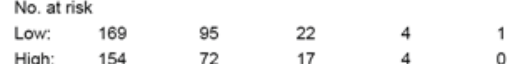

$F$

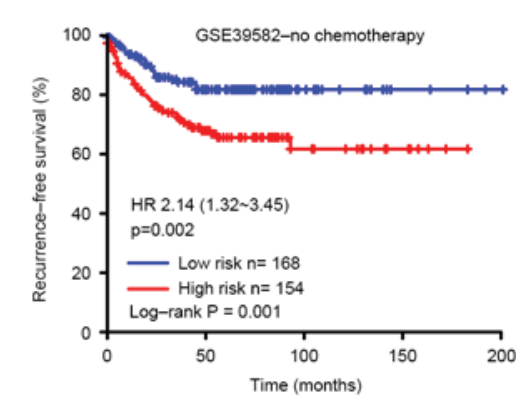

Figure 4. Kaplan-Meier estimates of the survival of patients receiving adjuvant chemotherapy using the eight-gene signature. (A) Kaplan-Meier estimator curves of OS for all patients ( $n=563$ ) in GSE39582 dataset. (B) Kaplan-Meier estimator curves of OS for patients receiving chemotherapy ( $n=240)$. (C) Kaplan-Meier estimator curves of OS for patients without chemotherapy ( $\mathrm{n}=323)$. (D) Kaplan-Meier estimator curves of RFS for all patients (n=561). (E) Kaplan-Meier estimator curves of RFS for patients with chemotherapy $(n=239)$. ( F) Kaplan-Meier estimator curves of RFS for patients without chemotherapy ( $n=322)$. Tick marks represent censored subjects. The survival differences between the high-risk and low-risk group were determined using the two-sided log-rank test. The number of patients at risk is listed below the survival curves. chemo, chemotherapy; HR, hazard ratio; OS, overall survival; RFS, recurrence-free survival.

A

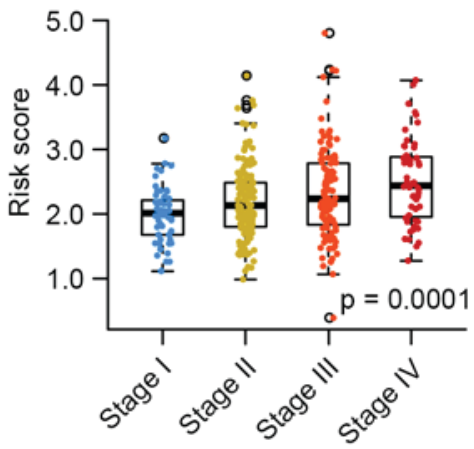

B

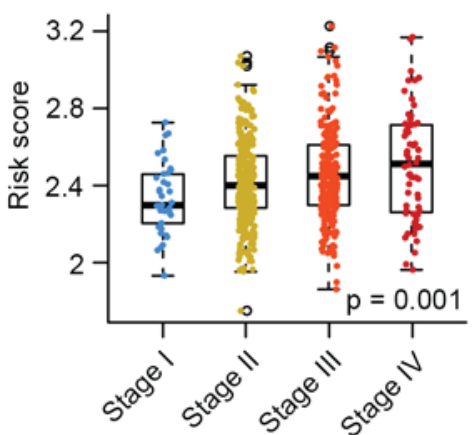

C

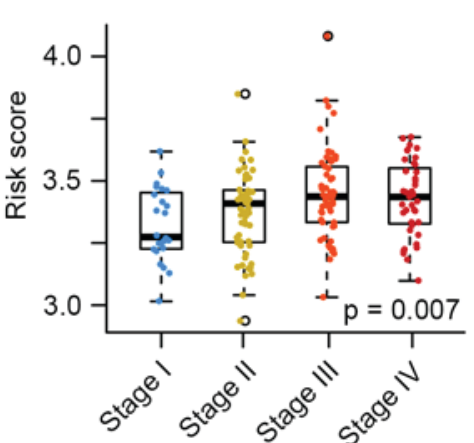

Figure 5. Risk score is associated with American Joint Committee on Cancer stage using two-way ANOVA. (A) In the TCGA set, the mean risk score increased with increasing tumor malignance ( $\mathrm{P}=0.0001$ ). (B) For GSE39582, the mean risk score increased with increasing tumor malignance ( $\mathrm{P}=0.001)$. (C) For GSE17536, the mean risk score increased with increasing tumor malignance $(\mathrm{P}=0.007)$. TCGA, The Cancer Genome Atlas.

disease-free survival prediction of the eight-gene signature model and the ColoGuideEx risk model for the GSE17536 dataset. The area under ROC (AUROC) was determined and compared between these two prognostic factors (Fig. 6). The ROC curves indicated that the AUROCs of the eight-gene signature and the ColoGuideEx were 0.698 and 0.582 , respectively $(\mathrm{P}=0.0801)$. These results indicated that the eight-gene signature model may possess increased predictive power compared with the ColoGuideEx model.

\section{Discussion}

The present study analyzed cancer stem cell signaling gene expression profiles from TCGA and the GEO database. By assessing the association between gene expression profiling and the clinical outcome of patients with colorectal cancer, the present study identified an eight-gene Hedgehog-Notch-Wnt signaling signature that was associated with the overall survival of the patients. The present study further validated 
Table II. Univariate and multivariate Cox regression analyses of overall survival in each data set.

\begin{tabular}{|c|c|c|c|c|c|c|}
\hline \multirow[b]{2}{*}{ Variables } & \multicolumn{3}{|c|}{ Univariate model } & \multicolumn{3}{|c|}{ Multivariate model } \\
\hline & HR & $95 \% \mathrm{CI}$ of $\mathrm{HR}$ & P-value & HR & $95 \% \mathrm{CI}$ of $\mathrm{HR}$ & P-value \\
\hline \multicolumn{7}{|l|}{ Training set (TCGA) $(\mathrm{N}=364)$} \\
\hline 8 gene risk score (high risk vs. low risk) & 2.38 & $1.46-3.87$ & 0.00048 & 2.19 & $1.27-3.76$ & 0.0045 \\
\hline Age $(\geq 65$ vs. $<65)$ & 1.85 & $1.11-3.09$ & 0.0183 & 3.04 & $1.68-5.49$ & 0.0002 \\
\hline Gender (male vs. female) & 1.52 & $0.94-2.47$ & 0.0903 & 1.28 & $0.77-2.14$ & 0.3465 \\
\hline Stage (I/II/III/IV) & 1.93 & $1.44-2.58$ & $1.00 \mathrm{E}-05$ & 1.91 & $1.40-2.61$ & $5.01 \mathrm{E}-05$ \\
\hline \multicolumn{7}{|l|}{ Testing set (GSE39582) (N=562) } \\
\hline 8 gene risk score (high risk vs. low risk) & 1.59 & $1.19-2.12$ & 0.0016 & 1.49 & $1.11-1.99$ & 0.0072 \\
\hline Age $(\geq 65$ vs. $<65)$ & 1.47 & $1.08-2.00$ & 0.0146 & 1.71 & $1.25-2.33$ & 0.0007 \\
\hline Gender (male vs. female) & 1.31 & $0.98-1.75$ & 0.0684 & 1.39 & $1.04-1.86$ & 0.0251 \\
\hline Stage $(\mathrm{I} / \mathrm{II} / \mathrm{III} / \mathrm{IV})$ & 1.92 & $1.57-2.34$ & $1.93 \mathrm{E}-10$ & 2.00 & $1.63-2.45$ & $2.95 \mathrm{E}-11$ \\
\hline \multicolumn{7}{|l|}{ Testing set (GSE17536) $(\mathrm{N}=177)$} \\
\hline 8 gene risk score (high risk vs. low risk) & 1.58 & $0.99-2.52$ & 0.0555 & 1.60 & $0.99-2.59$ & 0.0566 \\
\hline Age $(\geq 65$ vs. $<65)$ & 0.96 & $0.60-1.52$ & 0.848 & 1.33 & $0.81-2.17$ & 0.2483 \\
\hline Gender (male vs. female) & 1.10 & $0.69-1.76$ & 0.674 & 0.98 & $0.60-1.60$ & 0.9405 \\
\hline Stage $(I / I I / I I I / I V)$ & 2.85 & $2.11-3.86$ & $9.20 \mathrm{E}-12$ & 3.00 & $2.19-4.12$ & $1.02 \mathrm{E}-11$ \\
\hline \multicolumn{7}{|l|}{ Testing set (GSE17537) $(\mathrm{N}=55)$} \\
\hline 8 gene risk score (high risk vs. low risk) & 2.30 & $0.91-5.77$ & 0.0766 & 1.53 & $0.57-4.11$ & 0.3992 \\
\hline Age $(\geq 65$ vs. $<65)$ & 1.43 & $0.59-3.44$ & 0.425 & 1.86 & $0.73-4.78$ & 0.1958 \\
\hline Gender (male vs. female) & 0.68 & $0.28-1.66$ & 0.395 & 1.16 & $0.44-3.02$ & 0.7642 \\
\hline Stage (I/II/III/IV) & 2.97 & $1.56-5.65$ & 0.0009 & 2.98 & $1.54-5.78$ & 0.0012 \\
\hline
\end{tabular}

Bold values indicate statistical significance $\mathrm{P}<0.15$. TCGA, The Cancer Genome Atlas; HR, hazards ratio; $95 \%$ CI, $95 \%$ confidence interval.

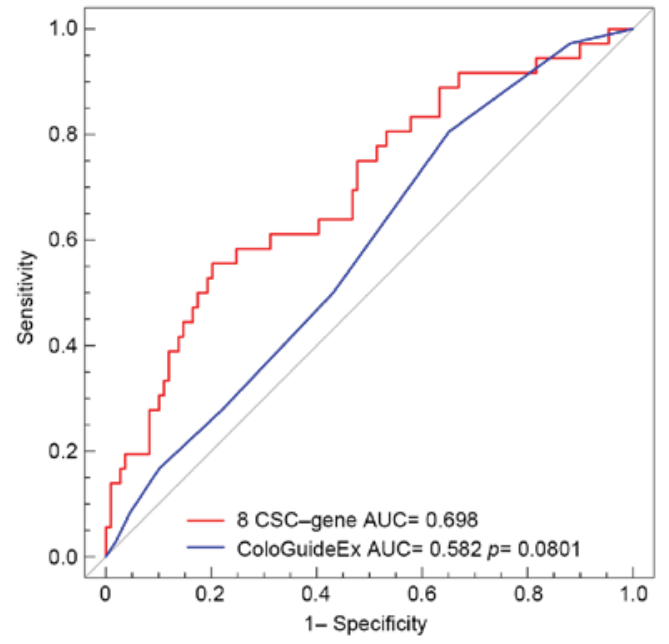

Figure 6. ROC analysis of the sensitivity and specificity of the eight-gene risk score and ColoGuideEx in predicting disease-free survival. For GSE17536, score performance was assessed by calculating the AUC of the eight-gene risk score and ColoGuideEx ( 0.698 and 0.582 , respectively; $\mathrm{P}=0.0801)$. ROC, receiver operating characteristic; CSC, cancer stem-like cell; AUC, area under the ROC curve.

the prognostic power of the eight-gene signature in independent cohorts and demonstrated that the eight-gene signature represents an independent predictor in colorectal cancer. This eight-gene signature requires further study in larger cohorts.
Characteristics of the eight CSC-associated genes. According to the predictive model of the present study, CCNE1 expression level was positive associated with good survival, whereas the expression of seven other genes were upregulated in the high-risk group compared with that in the low-risk group. CCNE1 has been reported as a tumor suppressor gene in multiple types of cancer (28). However, in the present study, high expression of CCNE1 was associated with increased survival in patients with colorectal cancer in TCGA and the GEO datasets (data not shown). The non-robustness could be due to the same gene functioning differently in different types or stages of cancer (29).

LRP2 is a component and an auxiliary Hedgehog signaling receptor (30). Andersen et al (31) reported that LRP2 regulated melanoma cell proliferation and survival rates, and that knocking down LRP2 induced apoptosis. LRP2 may serve a similar function in colorectal cancer. HEYL is regarded as a Notch effector, and upregulated HEYL expression has been reported in ovarian, breast and colon cancer (32). Han et al (33) demonstrated that HEYL was associated with Smad protein expression, transforming growth factor $\beta$ signaling, and initiating and progressing breast cancer. SFRP2 may augment Wnt16B signaling to promote a malignant phenotype and therapeutic resistance in the damaged tissue microenvironment, and increased SFRP2 expression has been reported to be associated with a poorer clinical outcome in colorectal cancer (34). LEF1, IGFBP3 and GADD45B, which were also reported to participate in stem cell signaling, have been reported to be 
upregulated in multiple types of cancer tissue, and possess growth-promoting functions in colon cancer cells (35-37). Cubilin was reported as a Wnt signaling target and modulator of the fibroblast growth factor (FGF) 8-FGF receptor signaling pathway, although this has not been demonstrated to be associated with a type of tumor (38).

The present study also performed a network analysis based on the eight signature genes in TCGA colorectal cancer cohorts downloaded from cBioPortal (see http://www. cbioportal.org/ for further pre-processing information). Default parameters were used to construct an interaction network of eight signature genes (data not shown). Certain well-studied genes in colorectal cancer, including APC, $\mathrm{B}$-Raf proto-oncogene, tumor protein $\mathrm{p} 53$, MYC proto-oncogene, RB transcriptional corepressor 1 and SMAD family member 4 exhibited closed interaction with the eight signature genes. Further study of the function of these genes is required to understand colorectal cancer tumorigenesis and development.

Eight-gene signature possesses prognostic power in patients with colorectal cancer undergoing adjuvant chemotherapy. Since the majority of the previous prognostic gene signatures were dominated by proliferation genes, the present study focused on cancer stem cell signaling pathways and provided novel insights into how cancer stem cell signaling contributes to colorectal cancer development. The eight-gene signature predicted unfavorable overall survival in those undergoing post-operative chemotherapy in the GSE39582 cohorts. Patients with an increased CSC-associated gene risk score were associated with adjuvant chemotherapy resistance. The present study proposed that this eight-gene signature reflects the activation state of tumor stem cell signaling, which may serve a crucial function in drug resistance and treatment outcome. Patients who have tumors with a high-risk CSC-associated gene signature could benefit from combined stem cell-targeted adjuvant therapy using this model, although further studies are required to verify this.

ROC and sensitivity. Further survival ROC analysis demonstrated that the eight-gene signature was comparable with the ColoGuideEx model $(\mathrm{P}=0.0801)$ for prognosis in GSE17536 sets, which were used in ColoGuideEx as the largest external validation datasets. Although the ColoGuideEx model is not typically used in deciding whether adjuvant chemotherapy is appropriate for a given patient with colorectal cancer (16), the test has been validated and rendered financially feasible for clinical practice using reverse transcription-PCR (39). The eight-gene signature of the present study could suffice in predicting the outcome of colorectal cancer, and predicts survival with increased accuracy compared with that predicted using ColoGuideEx.

Limitations of the present study. The limitations of the present study should be acknowledged. First, gene expression profile data were obtained from different platforms: TCGA for RNA-seq and GEO for microarray. Normalization methods differed between TCGA and GEO datasets. Secondly, only 334 cancer stem cell-associated genes were included in the present study, and the prognostic genes identified may not represent all cancer stem cell-associated gene candidates associated with overall survival in patients with colorectal cancer. Thirdly, more patient information and larger prospective patient cohorts are required to confirm the prognostic power of the eight-gene signature.

The present study developed a CSC-associated eight-gene signature that predicted colorectal cancer prognosis in four independent datasets. Further analysis revealed that the eight-gene signature may represent an independent prognostic factor with respect to age and stage. The eight-gene signature may assist molecular classification and outcome prediction in colorectal cancer. Further study is required to improve this eight-gene signature model in colorectal cancer.

\section{Acknowledgements}

The present study was supported by the National High Technology Research and Development Program of China (863 Program; grant no. 2015AA020104).

\section{References}

1. Siegel R, Naishadham D and Jemal A: Cancer statistics, 2012. Ca Cancer J Clin 62: 10-29, 2012.

2. Schneider M, Huber J, Hadaschik B, Siegers GM, Fiebig HH and Schüler J: Characterization of colon cancer cells: A functional approach characterizing CD133 as a potential stem cell marker. BMC Cancer 12: 96, 2012.

3. Asfaha S, Hayakawa Y, Muley A, Stokes S, Graham TA, Ericksen RE, Westphalen CB, von Burstin J, Mastracci TL, Worthley DL, et al: Krt19(+)/Lgr5(-) cells are radioresistant cancer-initiating stem cells in the colon and intestine. Cell Stem Cell 16: 627-638, 2015

4. Pang R, Law WL, Chu AC, Poon JT, Lam CS, Chow AK, Ng L, Cheung LW, Lan XR, Lan HY, et al: A subpopulation of CD26+ cancer stem cells with metastatic capacity in human colorectal cancer. Cell Stem Cell 6: 603, 2010.

5. Pattabiraman DR and Weinberg RA: Tackling the cancer stem cells-what challenges do they pose? Nat Rev Drug Discov 13: 497-512, 2014.

6. Varnat F, Siegl-Cachedenier I, Malerba M, Gervaz P and Ruiz i Altaba A: Loss of WNT-TCF addiction and enhancement of HH-GLI1 signalling define the metastatic transition of human colon carcinomas. EMBO Mol Med 2: 440-457, 2010.

7. Bao B, Wang Z, Ali S, Kong D, Li Y, Ahmad A, Banerjee S, Azmi AS, Miele L and Sarkar FH: Notch-1 induces epithelial-mesenchymal transition consistent with cancer stem cell phenotype in pancreatic cancer cells. Cancer Lett 307: 26-36, 2011.

8. Zhao C, Chen A, Jamieson CH, Fereshteh M, Abrahamsson A, Blum J, Kwon HY, Kim J, Chute JP, Rizzieri D, et al: Hedgehog signalling is essential for maintenance of cancer stem cells in myeloid leukaemia. Nature 458: 776-779, 2009.

9. Vermeulen L, De Sousa E Melo F, van der Heijden M, Cameron K, de Jong JH, Borovski T, Tuynman JB, Todaro M, Merz $\mathrm{C}$, Rodermond $\mathrm{H}$, et al: Wnt activity defines colon cancer stem cells and is regulated by the microenvironment. Nat Cell Biol 12: 468-476, 2015.

10. Takebe N, Miele L, Harris PJ, Jeong W, Bando H, Kahn M, Yang SX and Ivy SP: Targeting Notch, Hedgehog, and Wnt pathways in cancer stem cells: Clinical update. Nat Rev Clin Oncol 12: 445-464, 2015.

11. Takebe N, Harris PJ, Warren RQ and Ivy SP: Targeting cancer stem cells by inhibiting Wnt, Notch, and Hedgehog pathways. Nat Rev Clin Oncol 8: 97-106, 2011.

12. He J, Sheng T, Stelter AA, Li C, Zhang X, Sinha M, Luxon BA and Xie J: Suppressing Wnt signaling by the hedgehog pathway through sFRP-1. J Biol Chem 281: 35598-35602, 2006.

13. Clark-Langone KM, Sangli C, Krishnakumar J and Watson D: Translating tumor biology into personalized treatment planning: Analytical performance characteristics of the Oncotype DX colon cancer assay. BMC Cancer 10: 691, 2010. 
14. Gray RG, Quirke P, Handley K, Lopatin M, Magill L, Baehner FL, Beaumont C, Clark-Langone KM, Yoshizawa CN, Lee $\mathrm{M}$, et al: Validation study of a quantitative multigene reverse transcriptase-polymerase chain reaction assay for assessment of recurrence risk in patients with stage II colon cancer. J Clin Oncol 29: 4611-4619, 2011.

15. Salazar R, Roepman P, Capella G, Moreno V, Simon I, Dreezen C, Lopez-Doriga A, Santos C, Marijnen C, Westerga J, et al: Gene expression signature to improve prognosis prediction of stage II and III colorectal cancer. J Clin Oncol 29: 17-24, 2011.

16. Agesen TH, Sveen A, Merok MA, Lind GE, Nesbakken A, Skotheim RI and Lothe RA: ColoGuideEx: A robust gene classifier specific for stage II colorectal cancer prognosis. Gut 61: $1560-1567,2012$

17. Cline MS, Craft B, Swatloski T, Goldman M, Ma S, Haussler D and Zhu J: Exploring TCGA pan-cancer data at the UCSC cancer genomics browser. Sci Rep 3: 2652, 2013.

18. Lu X, Wan F, Zhang H, Shi G and Ye D: ITGA2B and ITGA8 are predictive of prognosis in clear cell renal cell carcinoma patients. Tumor Biol 37: 253-262, 2016.

19. Marisa L, Reyniès AD, Duval A, Selves J, Gaub MP, Vescovo L, Etienne-Grimaldi MC, Schiappa R, Guenot D, Ayadi M, et al: Gene expression classification of colon cancer into molecular subtypes: Characterization, validation, and prognostic value. PLoS Med 10: e1001453, 2013.

20. Smith JJ, Deane NG, Wu F, Merchant NB, Zhang B, Jiang A, Lu P, Johnson JC, Schmidt C, Bailey CE, et al: Experimentally derived metastasis gene expression profile predicts recurrence and death in patients with colon cancer. Gastroenterology 138 958-968, 2010

21. Freeman TJ, Smith JJ, Chen X, Washington MK, Roland JT, Means AL, Eschrich SA, Yeatman TJ, Deane NG and Beauchamp RD: Smad4-mediated signaling inhibits intestinal neoplasia by inhibiting expression of $\beta$-catenin. Gastroenterology 142: 562-571.e2, 2012

22. Luo T, Dunphy PS, Lina TT and McBride JW: Ehrlichia chaffeensis exploits canonical and noncanonical host wnt signaling pathways to stimulate phagocytosis and promote intracellular survival. Infect Immun 84: 686-700, 2015.

23. Simon R, Lam A, Li MC, Ngan M, Menenzes S and Zhao Y: Analysis of gene expression data using BRB-ArrayTools. Cancer Inform 3: 11-17, 2007.

24. Kawaguchi A, Iwadate Y, Komohara Y, Sano M, Kajiwara K, Yajima N, Tsuchiya N, Homma J, Aoki H, Kobayashi T, et al Gene expression signature-based prognostic risk score in patients with primary central nervous system lymphoma. Clin Cancer Res 18: 5672-5681, 2012.

25. Zhang XQ, Sun S, Lam KF, Kiang KM, Pu JK, Ho AS, Lui WM, Fung CF, Wong TS and Leung GK: A long non-coding RNA signature in glioblastoma multiforme predicts survival. Neurobiol Dis 58: 123-131, 2013.

26. Jin M, Li P, Zhang Q, Yang Z and Fu S: A four-long non-coding RNA signature in predicting breast cancer survival. J Exp Clin Cancer Res 33: 84, 2014.

27. Lan YT, Yang SH, Chang SC, Liang WY, Li AF, Wang HS, Jiang JK, Chen WS, Lin TC and Lin JK: Analysis of the seventh edition of American joint committee on colon cancer staging. Int J Colorectal Dis 27: 657-663, 2012.
28. Keyomarsi K, Tucker SL, Buchholz TA, Callister M, Ding Y, Hortobagyi GN, Bedrosian I, Knickerbocker C, Toyofuku W, Lowe $\mathrm{M}$, et al: Cyclin $\mathrm{E}$ and survival in patients with breast cancer. N Engl J Med 347: 1566-1575, 2002.

29. Pils D, Bachmayr-Heyda A, Auer K, Svoboda M, Auner V, Hager G, Obermayr E, Reiner A, Reinthaller A, Speiser P, et al: Cyclin E1 (CCNE1) as independent positive prognostic factor in advanced stage serous ovarian cancer patients-a study of the OVCAD consortium. Eur J Cancer 50: 99-110, 2014.

30. Christ A, Christa A, Kur E, Lioubinski O, Bachmann S, Willnow TE and Hammes A: LRP2 is an auxiliary SHH receptor required to condition the forebrain ventral midline for inductive signals. Dev Cell 22: 268-278, 2012.

31. Andersen RK, Hammer K, Hager H, Christensen JN, Ludvigsen M, Honoré B, Thomsen MB and Madsen M: Melanoma tumors frequently acquire LRP2/megalin expression, which modulates melanoma cell proliferation and survival rates. Pigment Cell Melanoma Res 28: 267-280, 2015.

32. Lu C, Bonome T, Li Y, Kamat AA, Han LY, Schmandt R, Coleman RL, Gershenson DM, Jaffe RB, Birrer MJ and Sood AK: Gene alterations identified by expression profiling in tumor-associated endothelial cells from invasive ovarian carcinoma. Cancer Res 67: 1757-1768, 2007.

33. Han L, Diehl A, Nguyen NK, Korangath P, Teo W, Cho S, Kominsky S, Huso DL, Feigenbaum L, Rein A, et al: The Notch pathway inhibits TGF $\beta$ signaling in breast cancer through HEYL-mediated crosstalk. Cancer Res 74: 6509-6518, 2014.

34. Sun Y, Zhu D, Chen F, Qian M, Wei H, Chen W and Xu J: SFRP2 augments WNT16B signaling to promote therapeutic resistance in the damaged tumor microenvironment. Oncogene 35: 4321-4334, 2016.

35. O'Connell MJ, Lavery I, Yothers G, Paik S, Clark-Langone KM, Lopatin M, Watson D, Baehner FL, Shak S, Baker J, et al: Relationship between tumor gene expression and recurrence in four independent studies of patients with stage II/III colon cancer treated with surgery alone or surgery plus adjuvant fluorouracil plus leucovorin. J Clin Oncol 28: 3937-3944, 2010.

36. Georges RB, Adwan H, Hamdi H, Hielscher T, Linnemann U and Berger MR: The insulin-like growth factor binding proteins 3 and 7 are associated with colorectal cancer and liver metastasis. Cancer Biol Ther 12: 69-79, 2011.

37. Hsieh TH, Hsu CY, Tsai CF4, Chiu CC, Liang SS, Wang TN, Kuo PL, Long CY and Tsai EM: A novel cell-penetrating peptide suppresses breast tumorigenesis by inhibiting $\beta$-catenin/LEF-1 signaling. Sci Rep 6: 19156, 2016.

38. Cases O, Perea-Gomez A, Aguiar DP, Nykjaer A, Amsellem S, Chandellier J, Umbhauer M, Cereghini S, Madsen M, Collignon J, et al: Cubilin, a high affinity receptor for fibroblast growth factor 8 , is required for cell survival in the developing vertebrate head. J Biol Chem 288: 16655-16670, 2013.

39. Park YY, Lee SS, Lim JY, Kim SC, Kim SB, Sohn BH, Chu IS, Oh SC, Park ES, Jeong W, et al: Comparison of prognostic genomic predictors in colorectal cancer. PLoS One 8: e60778, 2013. 